\title{
CAPACITIVE SENSE FEEDBACK CONTROL FOR MEMS BEAM STEERING MIRRORS
}

\author{
Baris Cagdaser, Anand Jog, Matt Last, Brian S. Leibowitz, Lixia Zhou, \\ Eric Shelton, Kristofer S.J. Pister, and Bernhard E. Boser \\ Berkeley Sensor \& Actuator Center \\ 497 Cory Hall, University of California at Berkeley, CA 94720
}

\begin{abstract}
This paper describes a feedback control system for a MEMS dual-axis scanner, which consists of two single-axis torsional mirrors. The mirror position is measured electronically with the sense capacitors and the EAM technique. The feedback system provides electronic damping and improves the dynamic performance of the high Q $(\sim 50)$ mirrors. Closed-loop controlled device achieves $400 \mathrm{~s}$ settling time. Due to the feed-through, drive signal mixes with the sense and limits the control bandwidth. Band-pass filtering and pseudo-differential sense attenuates the feed-through in the sense circuit.
\end{abstract}

\section{INTRODUCTION}

MEMS mirrors find their main application in the field of optical communication. In communication systems, MEMS mirrors serve as the beam steering device in a free space laser link $[1,2]$ or in an optical cross-connect $[3,4]$. In general, accuracy, speed, and large optical range are the main requirements for a mirror to be used in a communication system. Dielectric charging, poor mirror dynamics, or electrostatic pull-in are some of the reasons why MEMS mirrors fail to meet these requirements in open-loop electrostatic actuation. Closed-loop control can improve the limitations associated with the open-loop actuation. Previous closed-loop controlled mirrors have used the feedback for extending the drive range $[\mathbf{2 , 3}, \mathbf{4}]$. All of these devices employ the same dual-axis gimbaled structure, which uses parallel plate actuators for driving the mirror. Nonlinearities of the parallel plate actuation cause natural frequency down-tuning and pull-in resulting in limited angular range. Closed-loop control via feedback linearization [2,3] or sliding mode control [4] provides stable operation beyond the instability point.

Implementation of the feedback control requires position sense. Capacitive sense is the most commonly used method for position sensing in MEMS devices. In optical beam steering MEMS mirrors, position can also be measured by tracking the beam position by an external optical setup (usually a position sensing diode- PSD). Previous designs for optical cross-connects $[3,4]$ use optical measurements to implement the position sense. Capacitive sense has been implemented for only one design with a bigger mirror size [2]: $23 \mathrm{~mm}$ diameter compared to $900 \mathrm{~m} \mathrm{[3]}$, and $400 \mathrm{~m} \mathrm{[4]}$. The larger mirror diameter provides more room for the sense capacitor and improves the sensitivity.

Closed-loop control also improves the dynamic performance of a MEMS device. In this design, mirrors have vertical comb drive actuators [5]. Unlike parallel plate actuators, vertical comb drive is stable even at high drive voltages $(>100 \mathrm{~V},>20$ degrees optical) beyond which side instability ensues. This mirror design, however, has a high $\mathrm{Q}$ value ( $\sim 50$ in air) resulting in substantially underdamped step response. Feedback control is used for providing electronic damping. Position sensing is achieved by capacitive sense. Linear control techniques are enough to control the system over a 15 degrees optical steering range with the desired dynamic performance. The sense, drive, and the controller are all implemented using off-the-shelf components.

\section{DESIGN SUMMARY}

The MEMS scanner presented here is used as the beam steering device in a free-space optical communication system [1]. The acquisition, pointing, and beam stabilization requirements set the mirror specifications. Some key specifications are as follows:

20 degrees optical range for both axes

$<0.014$ degrees resolution ( $1 / 4$ of the laser beam)

$<1 \mathrm{~ms}$ settling time

Mirrors are fabricated in a multilevel beam SOI process [5]. A reflector deposited on the glass package lid optically couples the two single-axis mirrors. Figure 1 shows the scanner and a conceptual sketch of the laser path. As the first mirror (Y-axis scanner) rotates, the laser beam moves along the second mirror (Xaxis scanner). In order to meet the steering range requirement, $\mathrm{X}$ axis scanner is designed to be longer than the $\mathrm{Y}$-axis one. The $\mathrm{Y}$ mirror has a round reflector with $600 \mathrm{~m}$ diameter. The mirrors are 2 nd order systems with resonant frequencies of $1.7 \mathrm{kHz}(\mathrm{Y}-$ mirror) and $700 \mathrm{~Hz}$ (X-mirror).

In contrast to previous designs $[\mathbf{2 , 3 , 4}]$, this mirror lacks squeeze film damping resulting in high $\mathrm{Q}$ values even for operation in air. This high $\mathrm{Q}$ value results in substantial ringing and extremely long settling times. The damping ratio for an open loop driven mirror is $\sim 0.01$, which causes $100 \%$ overshoot and $\sim 600 \mathrm{msec}$ settling time (within $2 \%$ ) for the $700 \mathrm{~Hz} \mathrm{X}$-mirror. As mentioned earlier, feedback control provides the necessary damping for the system, and meets the goal of $<1 \mathrm{~ms}$ settling time. The feedback loop consists of 1) high voltage drive circuit with sufficiently large bandwidth $(0-100 \mathrm{~V}, 100 \mathrm{kHz}) ; 2)$ sense circuit using the electromechanical amplitude modulation (EAM) technique; 3) a phase lead controller for phase compensation.

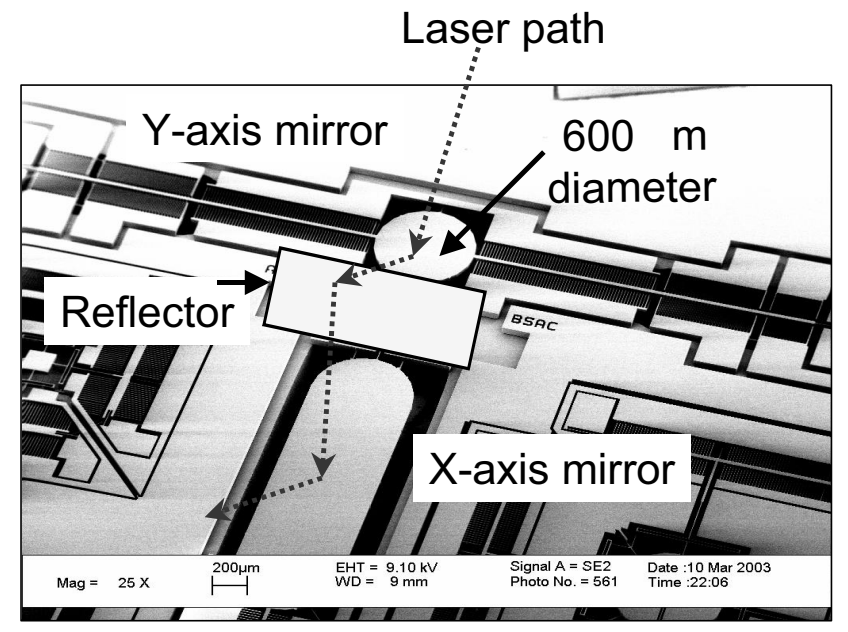

Figure 1. MEMS scanner with the sketch of optical coupling.

Travel support has been generously provided by the Transducers Reasearch Foundation and by the DARPA MEMS and DARPA BioFlips programs. 


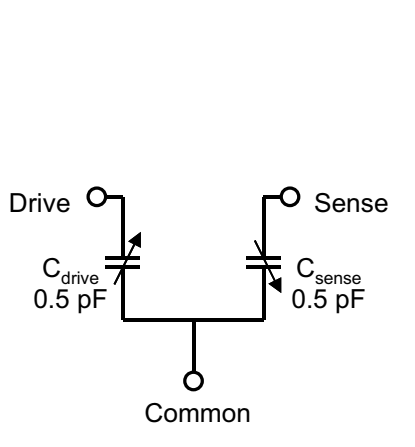

(a)

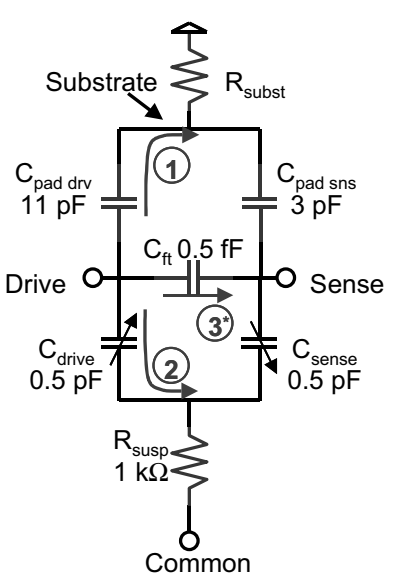

(b)
Figure 2. MEMS mirror electrical model a) ideal; b) actual with parasitics.

\section{SENSE CIRCUIT}

In closed-loop operation, the high voltage drive and the sense operate simultaneously. The sense circuit measures the mirror position while the drive is applying high voltage signals across the drive capacitor. Since drive and sense circuitry are connected to the same electrical network (MEMS), it is important to prevent drive and sense signal interference. This is achieved with the help of separate sense and drive capacitors. Since each capacitor has one plate connected to the MEMS, drive and sense capacitors share a common terminal, Figure 2 . In an ideal case, the common terminal is connected to a voltage supply that avoids interference between the drive and sense. In reality, however, there are parasitic effects that create feed-through paths for the drive signal. The three main parasitic effects are 1) finite conductivity of MEMS device layer; 2) parasitic pad capacitors; 3) package parasitics.

MEMS conductivity creates a problem because the common terminal of the sense and drive capacitor is attached to the MEMS. The only physical connection from MEMS to the bonding pads is through the torsion beam. Since torsion beams are long and thin structures, the electrical conductivity of the MEMS strongly determines the value of the parasitic resistor $\left(\mathrm{R}_{\text {susp }}\right)$. In this device, the high conductivity device layer $(1 \mathrm{~m} \Omega . \mathrm{cm})$ and a final metal deposition on the die minimize such effects. The 200-300A Al thin-film deposition also enhances the mirror optical reflectance.

Pads on the sides of the mirror suspend the stator fingers of the drive and sense capacitors. In the SOI process, the pads are separated from the substrate by a $2 \mathrm{~m}$ oxide layer. The process determines the minimum pad-substrate overlap. After minimizing the pad size, parasitic drive and sense capacitor values are $11 \mathrm{pF}$ $\left(\mathrm{C}_{\text {pad_drv }}\right)$ and $3 \mathrm{pF}\left(\mathrm{C}_{\text {pad_sns }}\right)$, Figure $2 \mathrm{~b}$. Since the connection to the substrate is nonideal ( $\left.\overline{\mathrm{R}}_{\text {subst }}\right)$, parasitic pad capacitors also create a feed-through path from the drive terminal to the sense.

The third type of feed-through is due to parasitics created during the packaging step. Closely spaced bond wires and package pins create feed-through paths between each other. In this case, the package parasitics between drive and sense $\left(\mathrm{C}_{\mathrm{ft}}, 0.5 \mathrm{pF}\right)$ dominates the other two feed-through mechanisms.

As explained above, the physical separation of sense and drive helps to attenuate the feed-through problem but is not a complete solution. In order to further decrease the interference we also separate the two signals in the frequency domain. In the electromechanical amplitude modulation (EAM) technique, a high frequency carrier $\left(\mathrm{V}_{\text {mod }}, 3 \mathrm{Mhz}\right)$ modulates the common node of the drive and sense capacitors. As the sense capacitor varies with mirror rotation, the amplitude of the resulting sense current also changes. In other words, position change modulates the amplitude of the sense current $\left(\mathrm{I}_{\mathrm{sns}}\right)$, Figure 3 . Due to this amplitude modulation, the sense signal $\left(\mathrm{V}_{\mathrm{sns}}\right)$ consists of a carrier and sense sidebands, Figure 4. In addition to these components, the low frequency drive signal $\left(\mathrm{V}_{\text {drv }}\right)$ also couples into the sense through three main parasitic paths discussed earlier.

In the EAM technique, demodulation is used to bring the sense signal down to the baseband. Ideally, this demodulation step modulates drive feed-through to the carrier frequency and brings the sense signal down to the base-band without mixing the two. Demodulator offset $\left(\mathrm{V}_{\text {offset }}\right)$, however, causes feed-through to leak into the base-band where it mixes with the sense signal, Figure 4.

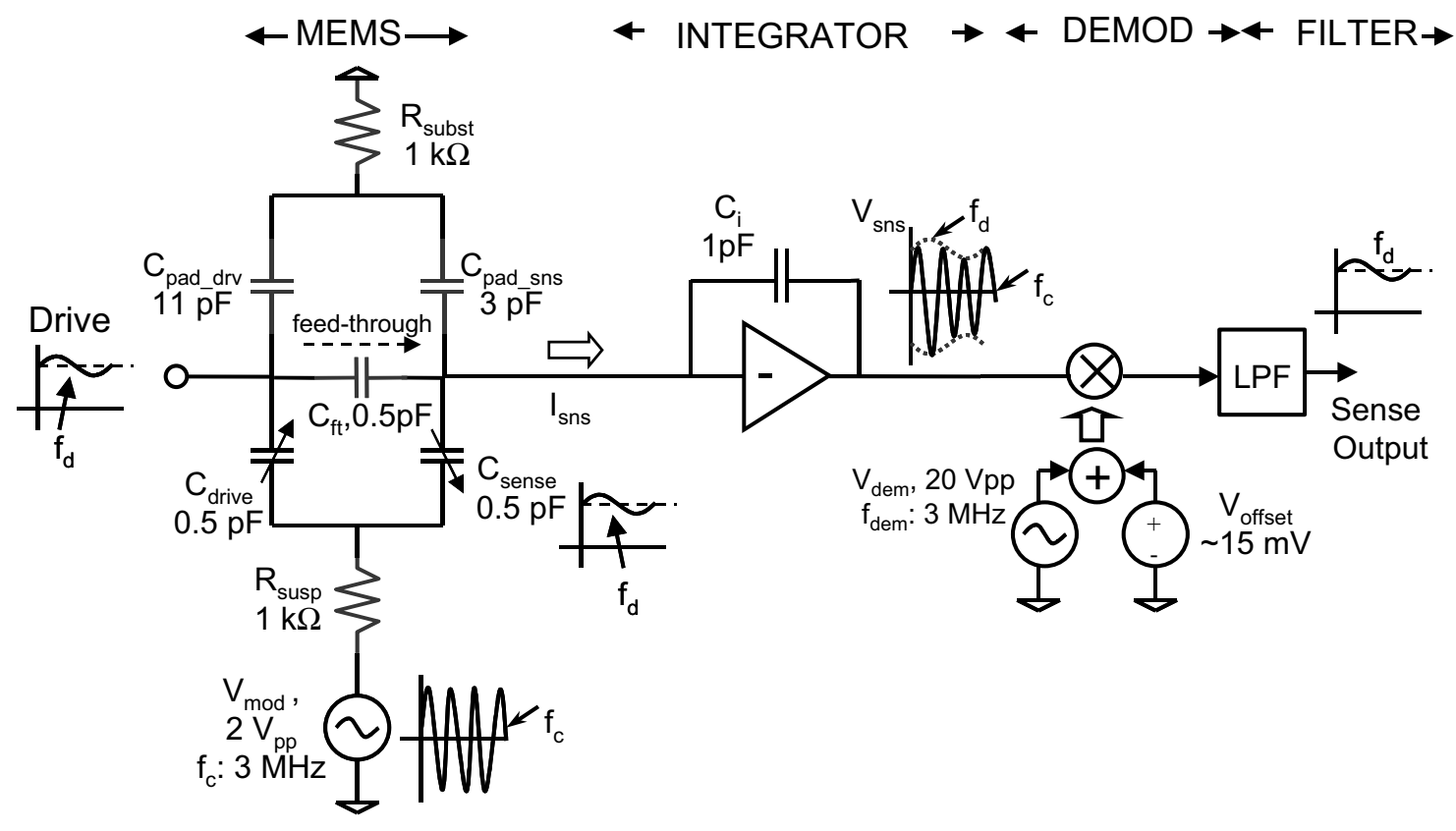

Figure 3. Capacitive sense with the EAM technique. 


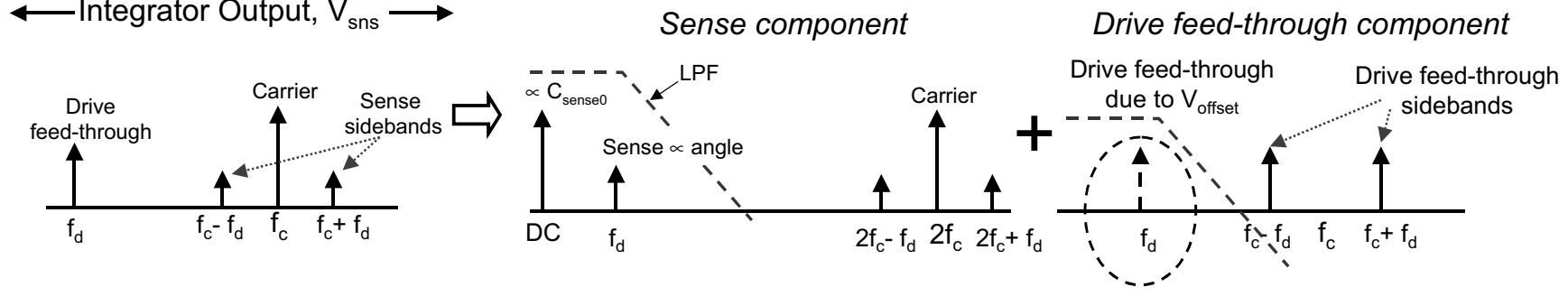

Figure 4. Modulation, demodulation, and the feed-through.

Demodulation is an analog multiplication step that multiplies the input signal by the demodulation signal. Consequently, multiplication by the offset is a direct path for the drive feedthrough to leak into the baseband and mix with the sense signal.

In order to reduce the feed-through, we use pseudodifferential sense and band-pass filtering, Figure 5. Band-pass filtering eliminates the low frequency drive signal before it leaks into the demodulator. Pseudo-differential sense helps the problem by increasing the sense signal strength. Sense signal is proportional to the modulation signal $\left(\mathrm{V}_{\text {mod }}\right)$ amplitude. Increasing the amplitude of the modulation signal eventually saturates the integrator circuit. In pseudo-differential sense, an external capacitor is connected to the input node of the integrator circuit. The external cancellation capacitor is driven by an out of phase version of the modulation signal resulting in the cancellation of the carrier that has no position information. The carrier amplitude is also much bigger than the sense sidebands. Using the pseudodifferential sense we can increase the modulation amplitude without saturating the integrator. As the sense signal becomes stronger, feed-through effects diminish. Using these circuit techniques, the sense bandwidth has improved by 10x, Figure 6 .

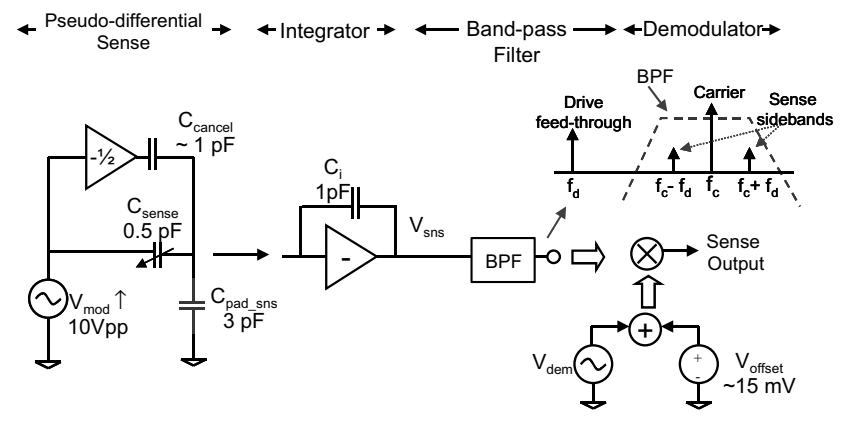

Figure 5. Bandpass filtering and pseudo-differential sense.

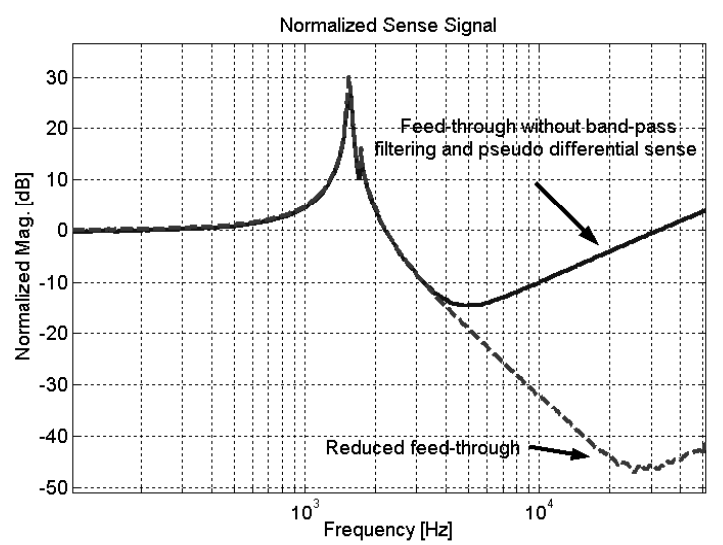

Figure 6. Feed-through before and after the circuit modifications.

\section{CONTROLLER DESIGN}

Phase compensation and the loop gain are the two main parameters in control loop design. Figure 7 shows the block diagram of the control system. The high voltage drive amplifier is in the gain stage. An analog phase lead filter implements the phase compensator. The main goal of the compensator is to provide enough phase margin for the stability of the feedback loop.

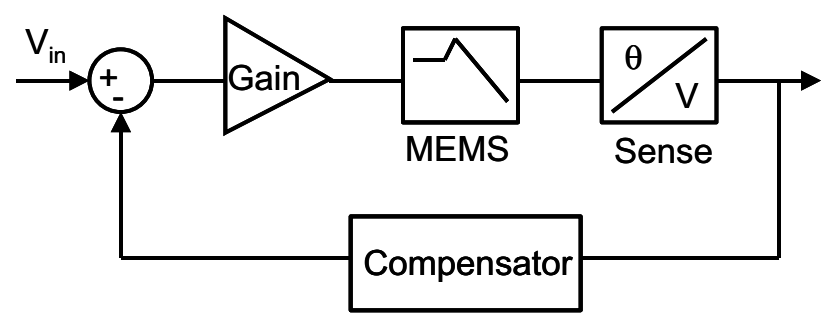

Figure 7. Closed-loop system block diagram.

Figure 8 shows the measured overall open-loop transfer function (OLTF) and the compensator. Increasing the loop gain improves the rejection of the disturbances. Higher loop gain also increases the unity gain crossover frequency $\left(\omega_{\mathrm{u}}\right)$ resulting in a need for phase compensation at higher frequencies. As long as we can provide enough phase, the closed-loop bandwidth also increases with the $\omega_{\mathrm{u}}$. In the control loop, the $2^{\text {nd }}$ order characteristics of the mirror causes a major 180 phase shift at the resonant frequency of the device. Considering that the other components in the loop also contribute to the phase delay, there is a practical limit for the maximum attainable closed-loop bandwidth.
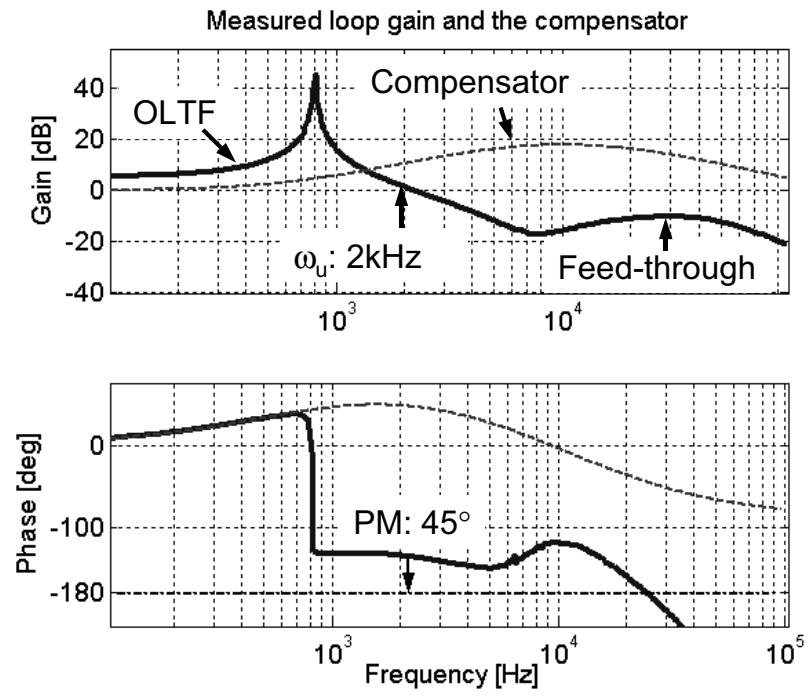

Figure 8. OLTF and the compensator. 
Ideally, the high voltage drive amplifiers are the slowest components in the loop (100kHz bandwidth) and limit the practical control bandwidth to $10-20 \mathrm{kHz}$. In practice, because the feedthrough effects begin to dominate at $\sim 20 \mathrm{kHz}$, we limit the loop gain by a low-pass filter at that frequency. After the filtering is applied the maximum closed-loop bandwidth of the system becomes $2-3 \mathrm{kHz}$ with a loop gain of $\sim 10 \mathrm{~dB}$ at DC.

\section{EXPERIMENTAL RESULTS}

The closed-loop system has been implemented on a PCB with off-the-shelf components. Dimensions of the two channel sense, controller, and drive board are $6 \mathrm{~cm} \times 12 \mathrm{~cm} \mathrm{x} 4 \mathrm{~cm}$. It weights 0.25 $\mathrm{lb}$, and the total power consumption is $5 \mathrm{~W}$, Figure 9 . The measurements shown here were done with the $700 \mathrm{~Hz} \mathrm{X}$-mirror. The controller has a zero at $580 \mathrm{~Hz}$, and two poles at $6.4 \mathrm{kHz}$ and $16 \mathrm{kHz}$. The second pole of the controller limits the compensator bandwidth in order to suppress the feed-through signal. The feedback system has a $2 \mathrm{kHz}$ bandwidth, Figure 10.

The electrostatic drive has nonlinearities primarily due to 1) torque (force) is proportional to the square of the drive voltage; 2 ) MEMS capacitors vary nonlinearly with the mirror position. The latter one also affects the sense. Consequently, mirror dynamics depend on the mirror position. The linear controller used in the system, however, is able to meet the settling time requirement $(<$ $1 \mathrm{~ms}$ ) for the range of 5 degrees to 20 degrees optical rotation. The best response is achieved at $50 \mathrm{~V}$ actuation ( 8 degrees) with $400 \mathrm{~s}$ settling time, Figure 11. The dynamic error for the settling time is the quarter beamwidth -0.014 degree $(0.25 \mathrm{mrad})$.

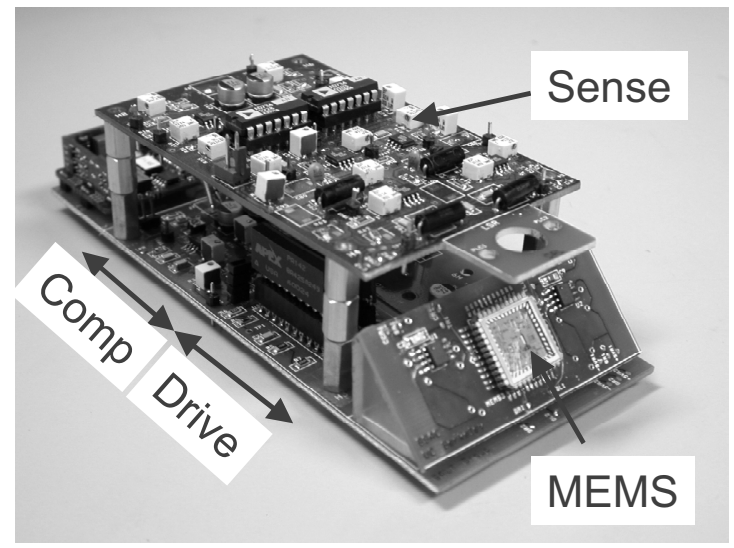

Figure 9. MEMS scanner and the control system.

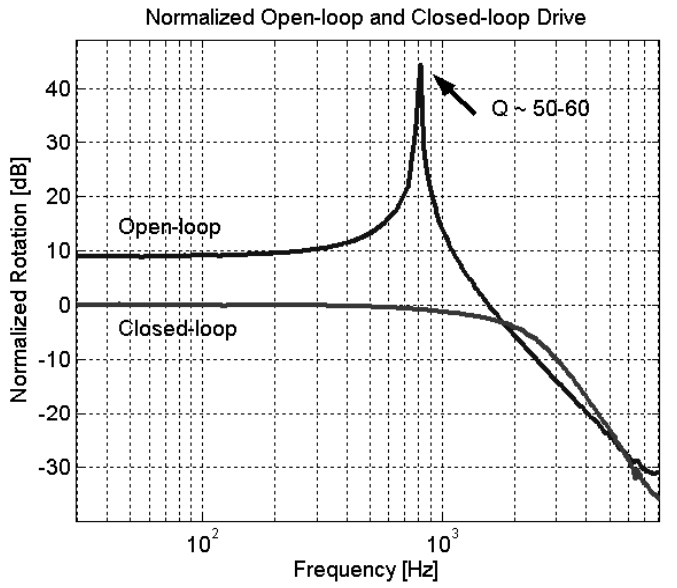

Figure 10. Measured closed-loop vs. open-loop actuation.

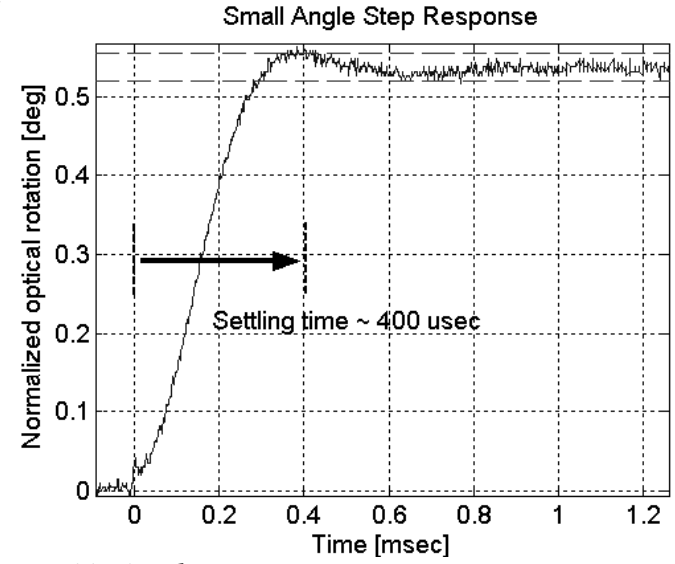

Figure 11. 0.5 degree step response.

The resolution of the closed-loop controlled single mirror is approximately 0.004 degree-rms (70 rad-rms). The dominant noise source is the demodulator in the sense circuit. The noise contribution of the rest of the system is less than half of the demodulator circuit.

\section{CONCLUSIONS}

Feedback control effectively improves the dynamic performance of MEMS mirrors. Minimizing parasitics and using the EAM technique attenuate the feed-through effects. Additional filtering introduced in the loop due to the feed-through limits the control bandwidth.

\section{ACKNOWLEDGEMENTS}

This research is funded by DARPA under the agreement DAAH01-00-C-0099.

\section{REFERENCES}

1. M. Last, B.S. Leibowitz, B. Cagdaser, A. Jog, L. Zhou, B. Boser, K.S.J. Pister, "Toward a wireless optical communication link between two small unmanned aerial vehicles," IEEE International Symposium on Circuits and Systems, vol.3, (2003), pp.930-933.

2. M. Suhonen et al, "Scanning micromechanical mirror for finepointing units of intersatellite optical links," Smart Materials \& Structures, vol.10, no.6, December (2001), pp.1204-1210.

3. N. Yazdi et al, "Robust sliding-mode control of electrostatic torsional micromirrors beyond the pull-in limit," Tranducers '03, Boston USA, vol. 2, June (2003), pp. 1450-1453.

4. T. Juneau et al, "Dual-axis mirror positioning using a nonlinear closed-loop controller," Tranducers '03, Boston USA, vol. 1, June (2003), pp. 560-563.

5. V. Milanović et al, "Monolithic High Aspect Ratio Two-axis Optical Scanner in SOI," Int. Conf. on Microelectromechanical Systems, MEMS2003, Kyoto Japan, January (2003), pp. 255-258.

6. V. Milanović, "Multilevel beam SOI-MEMS fabrication and applications," ICECS 2002 9th IEEE International Conference on Electronics, Circuits and Systems, vol.1, (2002), pp.281-5.

7. C. Nguyen, "Micromechanical signal processors," Ph.D. Dissertation, Dept. of EECS, University of California, Berkeley, (1994). 